\title{
Data report: clay mineralogical composition of northern Cascadia margin sediments, IODP Expedition $311^{1}$
}

\author{
Danièle Bartier, ${ }^{2}$ Marie-Madeleine Blanc-Valleron, ${ }^{2}$ Jean Marie Rouchy, ${ }^{2}$ and Catherine Pierre ${ }^{3}$
}

\section{Chapter contents}

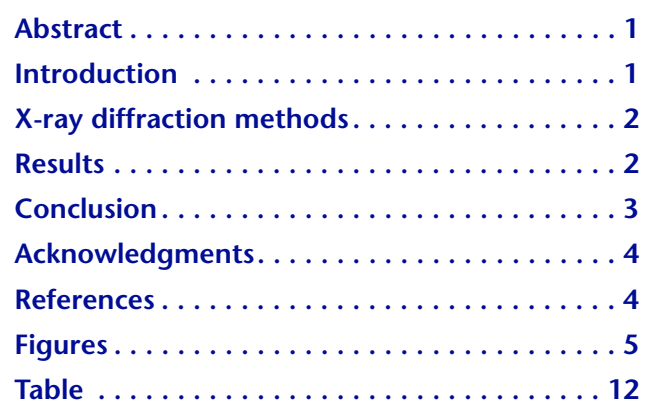

${ }^{1}$ Bartier, D., Blanc-Valleron, M.-M., Rouchy, J.M., and Pierre, C., 2008. Data report: clay mineralogical composition of northern Cascadia margin sediments, IODP Expedition 311. In Riedel, M., Collett, T.S., Malone, M.J., and the Expedition 311 Scientists, Proc. IODP, 311: Washington, DC (Integrated Ocean Drilling Program Management International, Inc.).

doi:10.2204/iodp.proc.311.208.2008

2UMR 5143 CNRS, Département Histoire de la Terre-Géologie, Muséum National d'Histoire Naturelle, 43 rue Buffon, 75005 Paris, France. Correspondence author: bartier@mnhn.fr ${ }^{3}$ CNRS-LOCEAN, Université Pierre et Marie Curie, 4 Place Jussieu, 75252 Paris Cedex 05, France.

\section{Abstract}

The clay mineralogical semiquantitative composition of 186 samples of hemipelagic sediments, turbiditic sediments, and authigenic carbonates from Integrated Ocean Drilling Program Sites U1325-U1329, northern Cascadia margin, were determined by Xray diffraction analyses. Oriented aggregates of clay-sized fractions were analyzed to estimate the relative percentages of smectite, illite, chlorite, kaolinite, and illite/vermiculite mixed layers. Illite/vermiculite mixed layers were observed for the first time in this area. Clay mineral assemblages show only modest long-term variations with no significant differences among Sites U1325-U1329. Variations in clay mineral assemblages are supposed to be mostly related to differences between hemipelagic sediments and turbiditic sediments. The sampling resolution and the age control are not good enough to define or recognize any climate control indicators in the clay mineral record.

\section{Introduction}

The Juan de Fuca plate is being subducted obliquely beneath the North American plate along the Washington, Oregon, and northern California continental margins. The Cascadia accretionary prism evolved in response to this oblique subduction. It is composed of folded and faulted abyssal plain turbidites and hemipelagic sediments (Kulm and Fowler, 1974; MacKay et al., 1992). In the northern section of the Cascadia margin, a gas hydraterelated bottom-simulating reflector occurs in a $30 \mathrm{~km}$ wide band parallel to the coast beneath much of the continental slope (Fig. F1A). Integrated Ocean Drilling Program Expedition 311 follows Ocean Drilling Program Legs 146 (north Hydrate Ridge) and 204 (south Hydrate Ridge) drilling campaigns in the Cascadia margin area to characterize gas hydrate processes. To constrain the distribution and concentration of gas hydrate in this region, four sites (U1326, U1325, U1327, and U1329) were drilled along a southwestnortheast transect during Expedition 311, from an uplifted ridge near the base of the continental slope (Site U1326) to near the edge of the continental shelf (Site U1329); a fifth site (U1328), representing a cold seep site with active fluid and gas flow, was also drilled (Fig. F1B) (see the "Expedition 311 Summary" chapter). This data report describes the clay assemblages identified by $\mathrm{X}$-ray diffraction of 186 turbiditic sediments, hemipelagic sedi- 
ments, and authigenic carbonates. The aim was originally to investigate potential modifications of clay mineral assemblages through sites, lithology, depth, and gas hydrate occurrence.

\section{X-ray diffraction methods}

\section{Sample preparation}

The sample preparation method used in this study is described in Holtzapffel (1985). Separation of claysized fractions $(<2 \mu \mathrm{m})$ started with a gentle crushing of samples and decalcification using a solution of $0.2 \mathrm{~N} \mathrm{HCl}$ in an Erlenmeyer flask. The preparation was washed with distilled water, and clay deflocculation was obtained through repeated centrifuging (2500 revolutions per minute [rpm] for $3 \mathrm{~min}$ ). After each centrifugation, water was eliminated and the plug was resuspended in distilled water. Generally, three to six centrifugation-suspension cycles were necessary until deflocculation. After transferring the suspended sediment to a glass beaker and vigorously shaking it, the clay fraction was separated by decantation using settling time based on Stoke's law. The extracted clay fraction was then centrifuged at 3500 rpm for $40 \mathrm{~min}$, and, finally, the clay plug was used to make an oriented clay glass slide. X-ray diffraction analyses were conducted on air-dried clay slides after saturation with ethylene glycol (at least $24 \mathrm{~h}$ ) and after heating at $490^{\circ} \mathrm{C}$ for $2 \mathrm{~h}$.

\section{X-ray diffraction parameters}

X-ray diffractograms were obtained at the Muséum National d'Histoire Naturelle of Paris using a Siemens D500 X-ray diffractometer with $\mathrm{CuK \alpha}$ radiation $(1.54 \AA)$ and $\mathrm{Ni}$ filter. Instrument parameters were set to $40 \mathrm{kV}$ accelerating voltage and $30 \mathrm{~mA}$ current. Scans were run from $2^{\circ}$ to $40^{\circ} 2 \theta$ with a scanning step size of $0.02^{\circ} 2 \theta$ and counting time of $4 \mathrm{~s}$. X-ray diagrams were studied using MacDiff (version 4.2.5) software (servermac.geologie.uni-frankfurt.de/Staff/Homepages/Petschick/RainerE.html) in order to establish the background line, smooth counts, correct peak positions (using quartz [100] peak at $4.24 \AA$ ), and compute integrated peak areas (total counts). The semiquantitative clay mineral proportions were estimated from the glycolated pattern. Clay mineral identification was made according to the position of the 001 series of basal reflections (Brown and Brindley, 1980). X-ray diffraction analyses allowed us to identify smectite, illite, chlorite, kaolinite, and some random mixed-layered illite/vermiculite $(\mathrm{I} / \mathrm{V})$. These $\mathrm{I} / \mathrm{V}$ mixed layers are characterized in air-dried conditions by a broad peak ranging from 10 to $13 \AA$, with a maximum intensity at $\sim 12 \AA$. This peak is not altered in position or intensity by glycol saturation but collapses to $10 \AA$ after heating (Fig. F2). In order to allow comparisons with published data from the northern Pacific region (Duncan et al., 1970; Karlin, 1980; Underwood, 2002; Underwood and Torres, 2006), the integrated areas were multiplied by weighting factors (Biscaye, 1965 ) and normalized to $100 \%$. Weighting factors are 4 for illite, 2 for kaolinite + chlorite, and 1 for smectite (Biscay, 1965); we chose to use 1 for I/V in order to compare results with those already published for this area (in which no I/V was detected), so percentages are only slightly "mathematically" modified. Chlorite and kaolinite were differentiated using the $3.54 \AA / 3.57 \AA$ peak ratio. The smectite term corresponds to a random R0 smectite/illite mixed layer (R0 I/S) and the amount of expandable layers was calculated based on the valley/peak height (v/p) ratio (after Biscay, 1965), the difference in the height of the base line from the high and low $2 \theta$ sides of the $5.2^{\circ} 2 \theta$ peak. Abacus allows consideration of the percentage of smectite portion from the measure of the $\mathrm{v} / \mathrm{p}$ parameter (the saddle-peak method; Rettke, $1981)$. The estimated analytical precision is $\pm 5 \%$.

\section{Results}

A total of 186 samples of hemipelagic samples, turbiditic samples, and carbonate concretion samples were analyzed in this study. Figures F3, F4, F5, F6, and F7 display downhole variations of clay minerals for each site. The X-ray diffraction data are illustrated in Table $\mathbf{T} 1$.

As previously stated, we used the factors of Biscay (1965) to estimate semiquantitative clay mineral percentages in order to compare our results with already published data. However, we must note that using these factors and 1 as the factor for I/V "minimizes" the proportion of I/V compared to semiquantitative estimates based on other methods described in Holtzapffel (1985). I/V mixed layers were never observed offshore Oregon (e.g., Karlin, 1980; Underwood, 2002; Gràcia et al., 2006; Underwood and Torres, 2006), whereas they are present in Pleistocene northwestern Atlantic sediments and relate to interglacial periods (e.g., Vanderaveroet et al., 2000). Vermiculite and random mixed-layer minerals were also described in late Pleistocene sediments of Vancouver Island (Blaise, 1989). The expandability values in R0 $\mathrm{I} / \mathrm{S}$ for each site are quite similar (mean $=\sim 60 \%-$ $65 \%$; lower values are obviously observed in smec- 
tite-poor samples). Therefore, as with Underwood and Torres (2006), we suppose that the expandable clay is probably detrital in origin with small amounts of interlayered illite.

\section{Site U1326}

Site U1326 is located on top of the first uplifted ridge of accreted sediments at the far western downslope end of the transect. Thirty-three samples from lithostratigraphic Unit I (Hole U1326C), Unit II (Holes U1326C and U1326D), and Unit III (Hole U1326D) were analyzed (Table T1; Fig. F3). Three samples analyzed for Unit I show a similar clay assemblage composed of smectite (19\%), illite (42\%), chlorite (28\%), and kaolinite $(12 \%)$. There is an anticorrelation between smectite and illite in Unit II and between illite and chlorite (+ kaolinite) in Unit III. Moreover, as noted by Bahr et al., there is a trend toward higher illite and chlorite (+ kaolinite) contents in the lower part of Unit II and all of Unit III. The lower smectite content in Unit III is accompanied by a slight decrease in the amount of expandable layers (Percent expand in Table T1). The authigenic carbonate-rich sample at 235.90 meters below seafloor (mbsf) does not show any particular clay content.

\section{Site U1325}

Site U1325 is located within the first slope basin in the southwestern part of the transect. Thirty-five samples from lithostratigraphic Units IA, IB, II, and III (Hole U1325B) and Unit IV (Hole U1325C) were analyzed. As for Site U1326, clay mineral assemblages are composed of smectite, illite, chlorite, kaolinite, and I/V (Table T1; Fig. F4). No global trend was noted except in Units I-II, in which the observed moderate anticorrelation between smectite and illite could be linked to sediment grain size, turbiditic samples being more illitic, whereas hemipelagic samples are more smectitic. Unit IV (as Unit III for Site U1326) appears slightly richer in chlorite and I/V than Units I-III and poorer in smectite. The authigenic carbonate-rich sample analyzed in Unit III does not show any particular clay content.

\section{Site U1327}

Site U1327 is located on the midcontinental slope off Vancouver Island, several hundred meters from Site 889 (Leg 146). Forty-two samples from lithostratigraphic Unit I (Hole U1327C), Unit II (Holes U1327C and U1327D), and Unit III (Hole U1327C) were analyzed (Table T1; Fig. F5). Units I-III display an anticorrelation between smectite and illite related to sediment grain size. Unit III is slightly richer in illite and poorer in smectite than Units I and II, whereas Unit I displays a little more I/V. Six authigenic carbonate-rich samples were analyzed (one in Unit I, four in Unit II, and one in Unit III) with no particular clay content.

\section{Site U1328}

Site U1328 is located $3.7 \mathrm{~km}$ southeast of Site U1327 on the midcontinental slope off Vancouver Island. Forty-four samples from lithostratigraphic Unit I (Holes U1328B and U1328C), Unit II (Hole U1328C), and Unit III (Hole U1328C) were analyzed (Table T1; Fig. F6). As for the precedent sites, an anticorrelation trend between smectite and illite, related to sediment grain size, was observed. The most smectitic samples (and poorest in illite) are observed at the base of Unit I between 105 and 130 mbsf. Lithostratigraphic Unit II is slightly richer in illite and poorer in smectite than Units I and III. Four authigenic carbonate-rich samples were analyzed in Unit III with no particular clay content.

\section{Site U1329}

Site U1329 is the easternmost and shallowest site of the transect. Moreover, it is the only site to display Miocene sediments occurring in discordance below Pleistocene sediments. Twenty-seven samples from lithostratigraphic Units I-III (Hole U1328C) were analyzed (Table T1; Fig. F7). From 0.74 to 35.35 mbsf the clay content of samples is characterized by the lack of kaolinite (all of Unit I and the first sample of Unit II). Lithostratigraphic Unit I appears also slightly poorer in smectite than Units II and III. Globally, an anticorrelation trend between smectite and illite fractions is also observed in the three units. Compared to Pleistocene sediments, Miocene sediments are slightly richer in smectite and kaolinite and poorer in illite and chlorite.

\section{Conclusion}

At Sites U1325-U1329 of Expedition 311, the clay mineral assemblages are composed of smectite, illite, chlorite, kaolinite, and illite-vermiculite (I/V) mixedlayers. I/V layers have not been observed in the Cascadia margin area (e.g., Karlin, 1980; Underwood and Torres, 2006), whereas they are present in Pleistocene northwestern Atlantic sediments (e.g., Vanderaveroet et al., 2000) and are related to interglacial periods. However, in this study, the sampling resolution and the age control are not good enough to de- 
fine or recognize any climate control in the clay mineral record.

Clay mineral variations are slight and probably partly due to the natural heterogeneity of the claysized sediment budget. Nevertheless, it is possible to see some differences between sites and between units in the same site. Lithostratigraphic Unit I at Site U1329 does not contain kaolinite. The lowest smectite contents are observed in lithostratigraphic Unit IV at Site U1325 and Unit III at Site U1326, whereas higher values are recorded in the Miocene of lithostratigraphic Unit III at Site U1329. In all sites, a global anticorrelation trend between illite and smectite fractions is observed. This trend could be correlated to sediment grain size, hemipelagic sediments being slightly richer in smectite and turbiditic samples slightly enriched in illite. In the samples studied, there is no evidence of diagenetic impact of gas hydrate and/or carbonate diagenesis occurrence on the clay mineral fraction.

\section{Acknowledgments}

The authors would like to thank Pierre Clément and Anne-Marie Brunet (MNHN, Paris, France) for help with sample processing and XRD analyses and Liviu Giosans and editor Lorri Peters for their careful review of the manuscript. This research used samples and/or data provided by the Integrated Ocean Drilling Program (IODP). Funding for this research was provided by an Eclipse II grant of the CNRS.

\section{References}

Biscaye, P.E., 1965. Mineralogy and sedimentation of recent deep-sea clay in the Atlantic Ocean and adjacent seas and oceans. Geol. Soc. Am. Bull., 76(7):803-831. doi:10.1130/0016-

7606(1965)76[803:MASORD]2.0.CO;2

Blaise, B., 1989. Clay-mineral assemblages from Late Quaternary deposits on Vancouver Island, southwestern British Columbia, Canada. Quat. Res., 31(1):41-56. doi:10.1016/0033-5894(89)90084-7

Brown, G., and Brindley, G.W., 1980. X-ray diffraction procedures for clay mineral identification. In Brindley, G.W., and Brown, G. (Eds.), Crystal Structures of Clay Minerals and Their X-ray Identification. Mineral. Soc. Monogr. London, 5:305-359.
Duncan, J.R., Kulm, L.D., and Griggs, G.B., 1970. Clay mineral composition of late Pleistocene and Holocene sediments of Cascadia Basin, northeastern Pacific Ocean. J. Geol., 78:213-221.

Gràcia, E., Martínez-Ruiz, F., Piñero, E., Larrasoaña, J.C., Vizcaino, A., and Ercilla, G., 2006. Data report: grain-size and bulk and clay mineralogy of sediments from the summit and flanks of southern Hydrate Ridge, Sites 1244-1250, ODP Leg 204. In Tréhu, A.M., Bohrmann, G., Torres, M.E., and Colwell, F.S. (Eds.), Proc. ODP, Sci. Results, 204: College Station, TX (Ocean Drilling Program), 1-19. doi:10.2973/odp.proc.sr.204.110.2006

Holtzapffel, T., 1985. Les minéraux argileux: préparation, analyse diffractométrique et détermination. Publ. Soc. Geol. Nord., 12.

Karlin, R., 1980. Sediment sources and clay mineral distributions off the Oregon coast. J. Sediment. Petrol., 50:543-560.

Kulm, L.D., and Fowler, G.A., 1974. Oregon continental margin structure and stratigraphy: a test of the imbricate thrust model. In Burke, C.A., and Drake, C.L. (Eds.), The Geology of Continental Margins: New York (Springer), 261284.

MacKay, M.E., Moore, G.F., Cochrane, G.R., Moore, J.C., and Kulm, L.D., 1992. Landward vergence and oblique structural trends in the Oregon margin accretionary prism: implications and effect on fluid flow. Earth Planet. Sci. Lett., 109(3-4):477-491. doi:10.1016/0012821X(92)90108-8

Rettke, R.C., 1981. Probable burial diagenetic and provenance effects on Dakota Group clay mineralogy, Denver Basin. J. Sediment. Petrol., 51:541-551.

Underwood, M., and Torres, M., 2006. Data report: composition of clay minerals from hemipelagic sediments at Hydrate Ridge, Cascadia subduction zone. In Tréhu, A.M., Bohrmann, G., Torres, M.E., and Colwell, F.S. (Eds.), Proc. ODP, Sci. Results, 204: College Station TX (Ocean Drilling Program), 1-15. doi:10.2973/odp.proc.sr.204.127.2006

Underwood, M.B., 2002. Strike-parallel variations in clay minerals and fault vergence in the Cascadia subduction zone. Geology, 30(2):155-158. doi:10.1130/00917613(2002)030<0155:SPVICM>2.0.CO;2

Vanderaveroet, P., Bout-Roumazeilles, V., Fagel, N., Chamley, H., and Deconinck, J.F., 2000. Significance of random illite-vermiculite mixed layers in Pleistocene sediments of the northwestern Altlantic Ocean. Clay Miner., 35(4):679691. doi:10.1180/000985500547133

Initial receipt: 26 June 2008

Acceptance: 5 September 2008

Publication: 17 November 2008

MS 311-208 
Figure F1. A. Plate tectonic setting of the Cascadia margin with general location of the drilling transect near previous ODP Sites $889 / 890$. A bottom-simulating reflector is present on $\sim 50 \%$ of the midcontinental slope, as denoted by the shaded area (see the "Expedition 311 summary" chapter). B. Multibeam bathymetry map along the transect across the accretionary prism, showing the location of the drilling transect (Sites U1326, U1325, U1327, and U1329), cold vent Site U1328, and multichannel seismic (MCS) Line 89-08 (Courtesy of D. Kelley, J. Delaney, and D. Glickson, University of Washington; C. Barnes, C. Katnick, Neptune Canada, University of Victoria; funded by the University of Washington and the W.M. Keck Foundation).

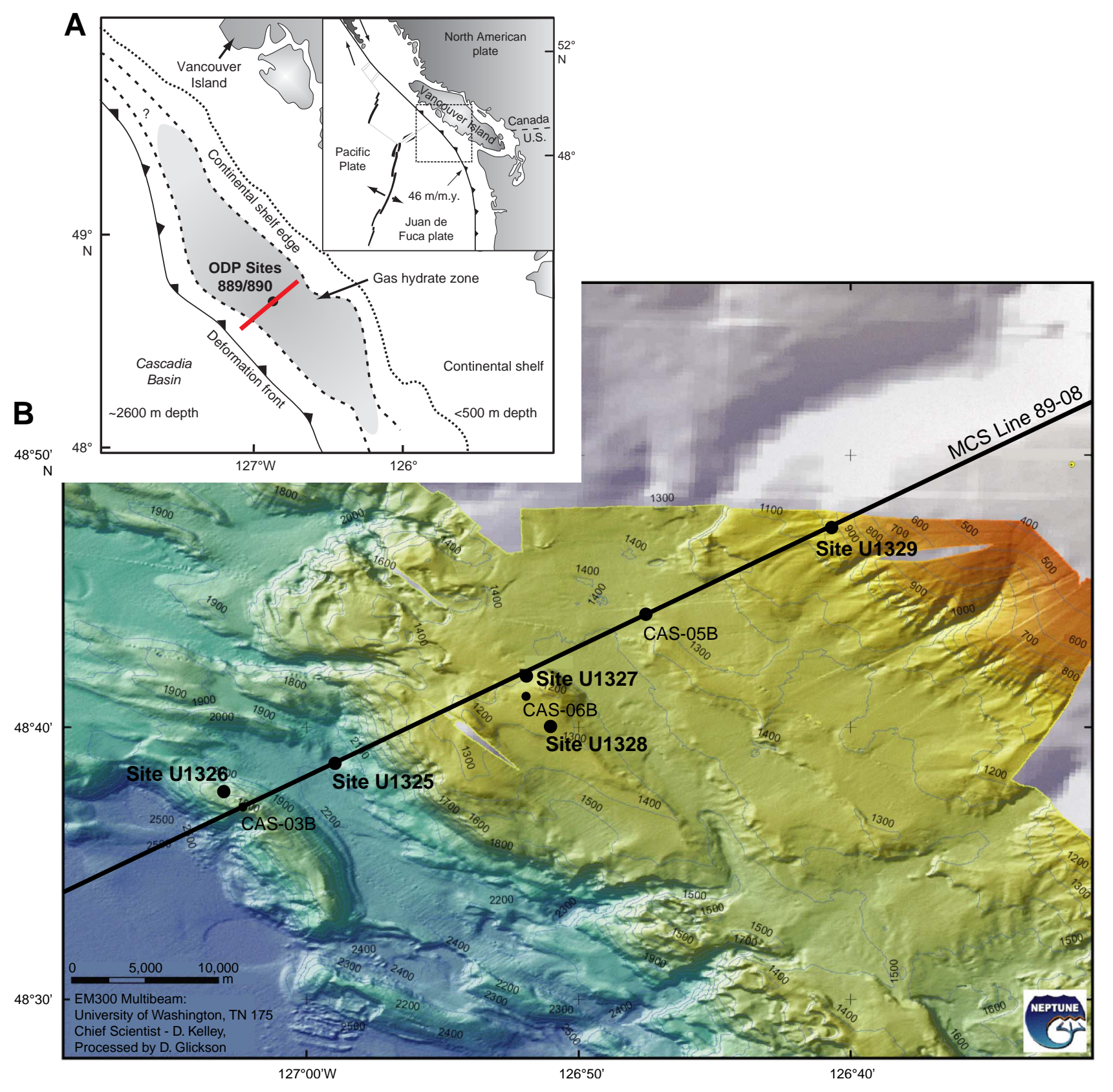


Figure F2. Selected X-ray diffractograms of Sample 311-U1326D-8X-3, 75-77 cm, showing the presence of illite/vermiculite $(\mathrm{I} / \mathrm{V})$ in the clay size fraction. $\mathrm{Sm}=$ smectite, $\mathrm{Chl}=$ chlorite, $\mathrm{Ill}=$ illite, $\mathrm{Amp}=$ amphibole, Kaol = kaolinite, Fd = feldspar, Qtz = quartz.

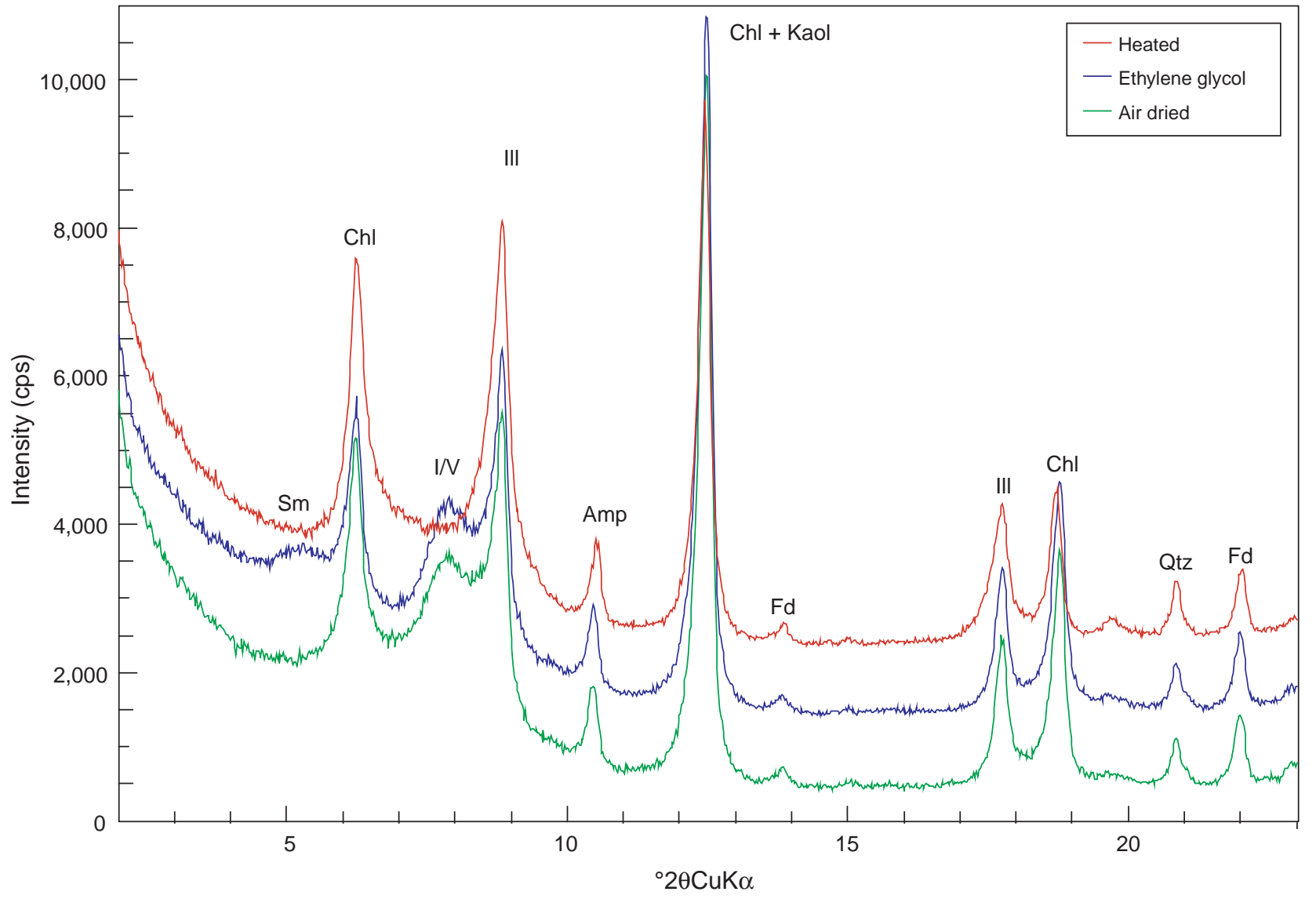


Figure F3. Downhole variations of clay minerals in sediment and authigenic carbonate samples, Site U1326. BGHSZ = bottom gas hydrate stability zone. $\mathrm{I} / \mathrm{V}=$ illite/vermiculite.

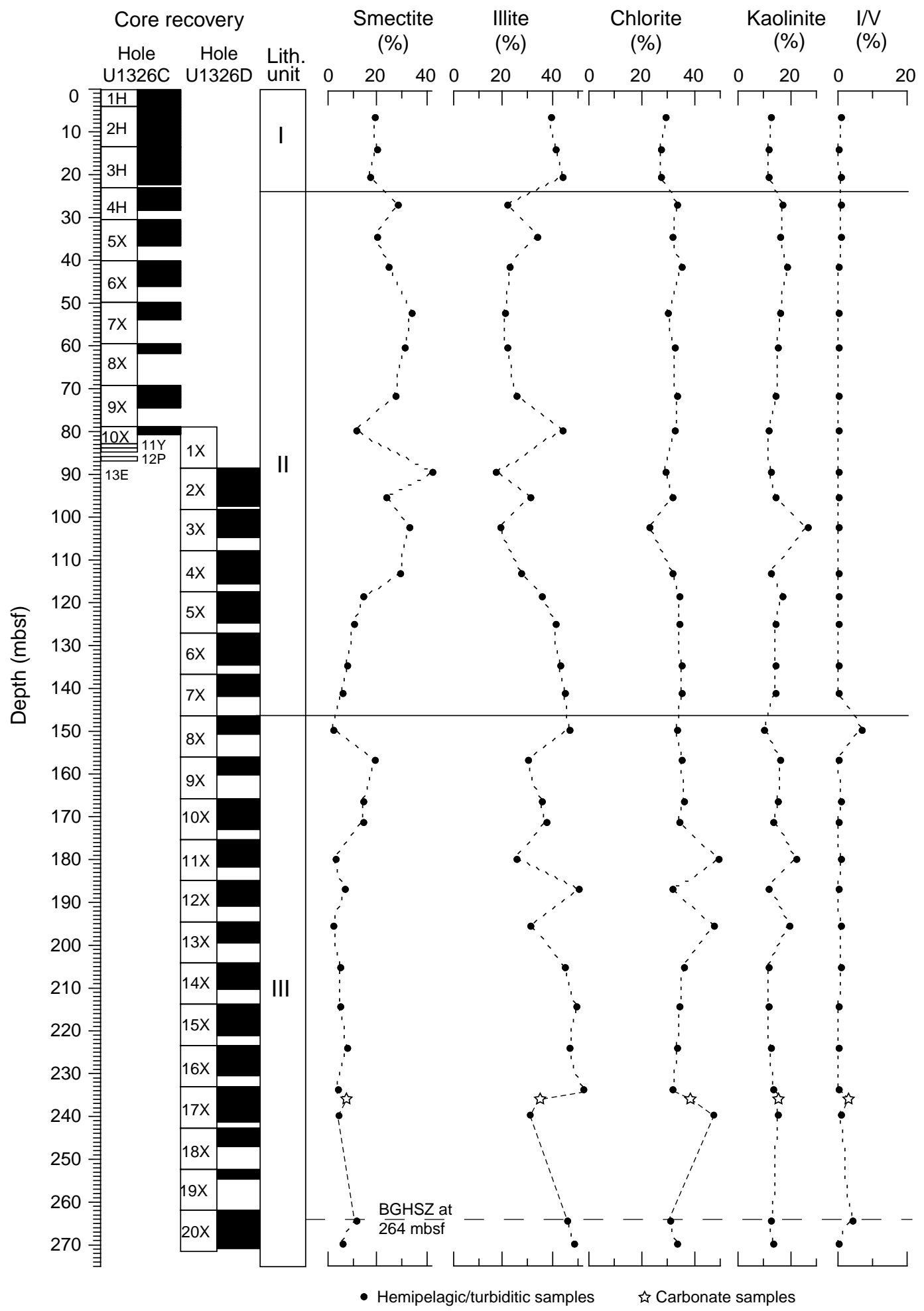


Figure F4. Downhole variations of clay minerals in sediment and authigenic carbonate samples, Site U1325. BGHSZ = bottom gas hydrate stability zone. $\mathrm{I} / \mathrm{V}=$ illite/vermiculite.

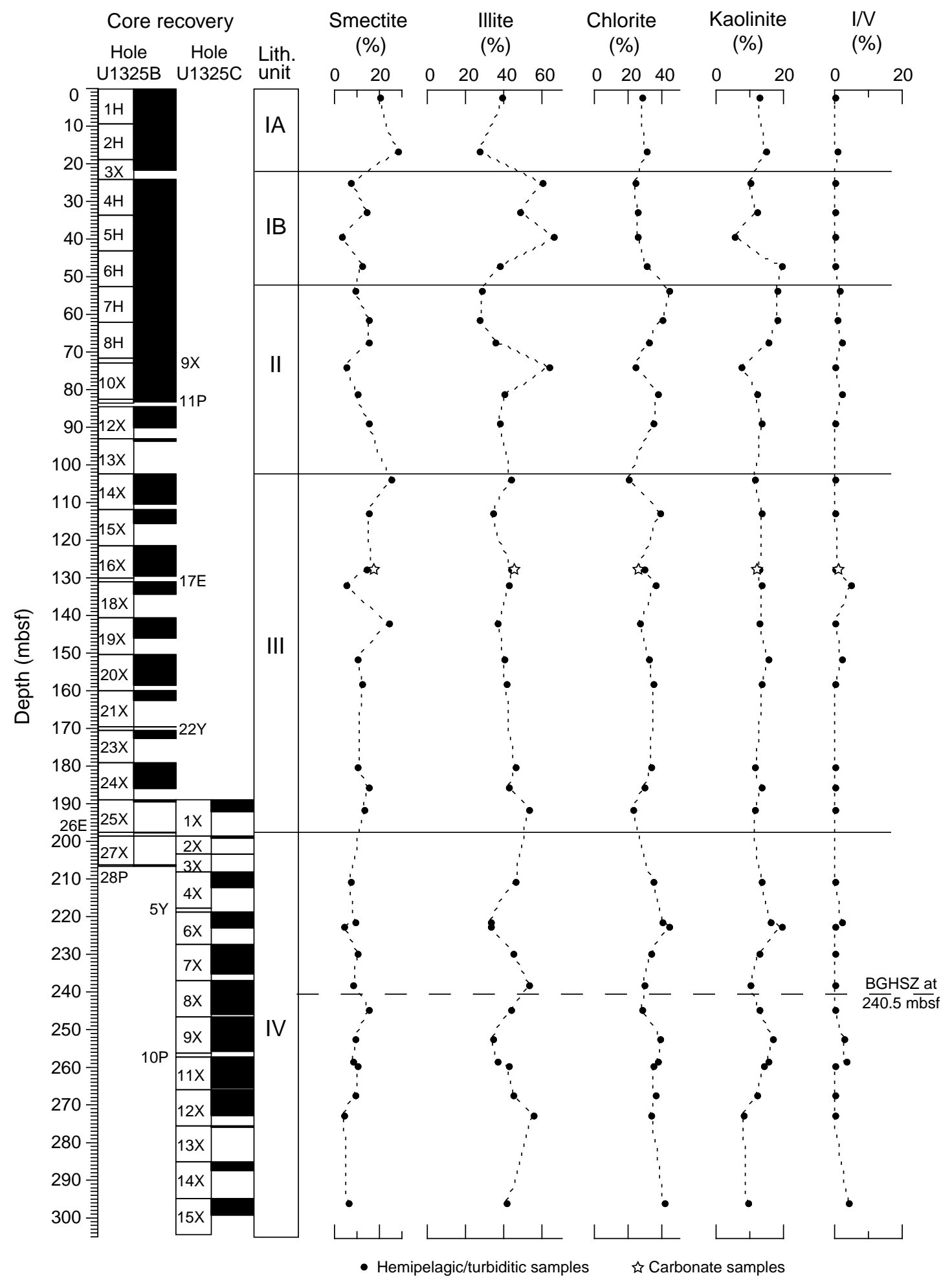


Figure F5. Downhole variations of clay minerals in sediment and authigenic carbonate samples, Site U1327. BGHSZ = bottom gas hydrate stability zone. $\mathrm{I} / \mathrm{V}=$ illite/vermiculite.

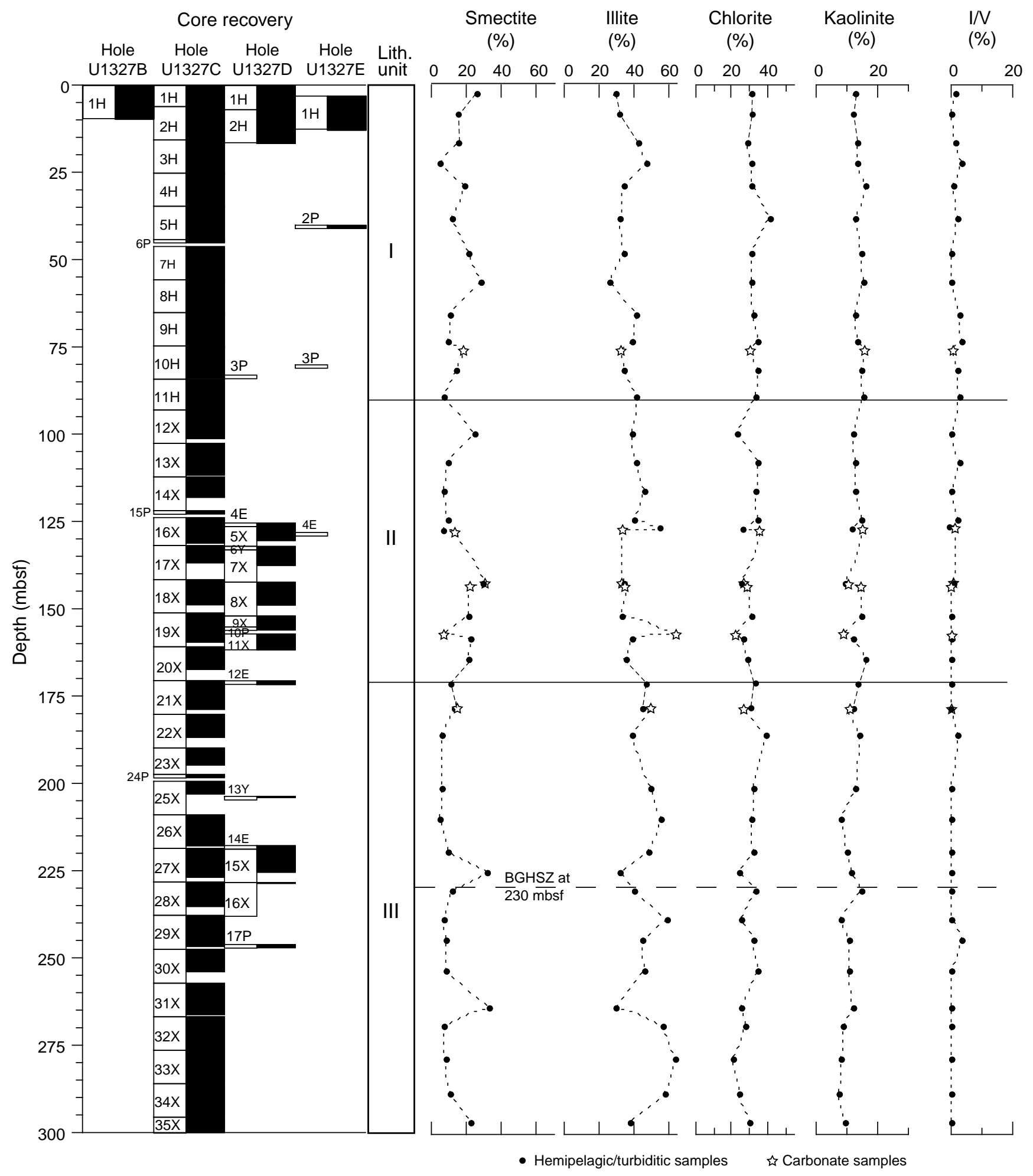


Figure F6. Downhole variations of clay minerals in sediment and authigenic carbonate samples, Site U1328. BGHSZ = bottom gas hydrate stability zone. $\mathrm{I} / \mathrm{V}=$ illite/vermiculite .

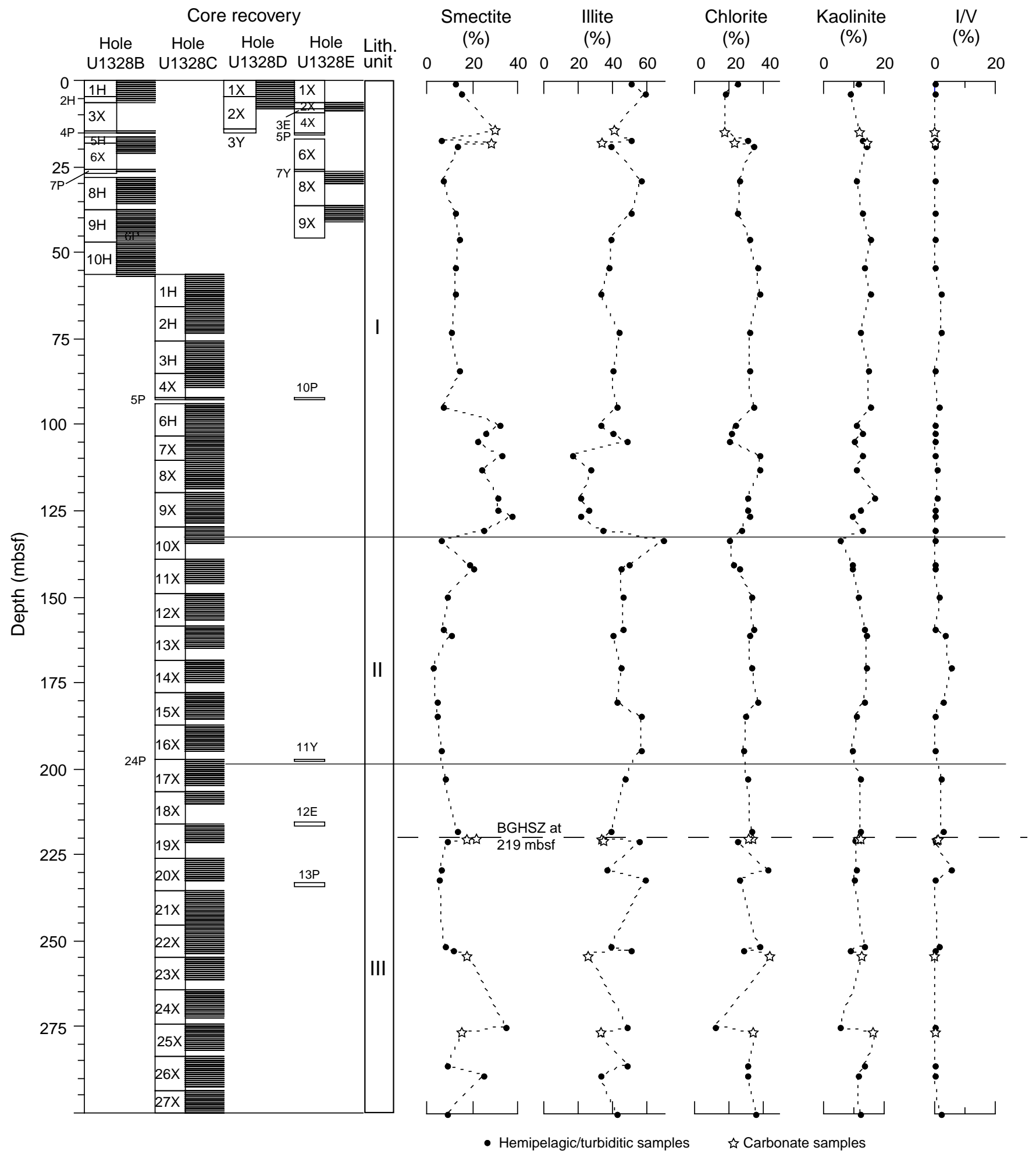


Figure F7. Downhole variations of clay minerals in sediment, Site U1329. BGHSZ = bottom gas hydrate stability zone. $\mathrm{I} / \mathrm{V}=$ illite/vermiculite.

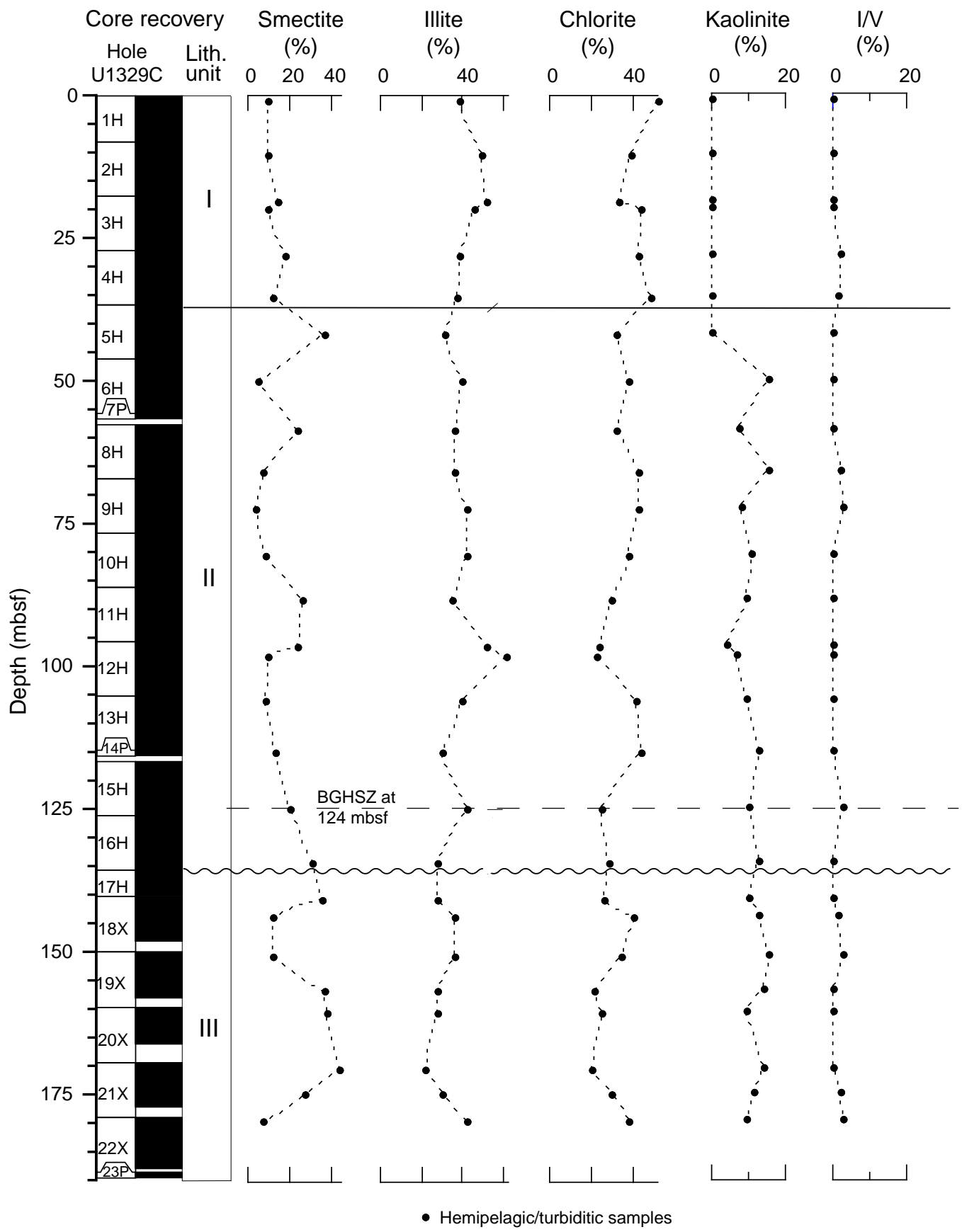


Table T1. X-ray diffractometry mineralogical results of clay fraction $(<2 \mu \mathrm{m})$. (See table note.) (Continued on next four pages.)

\begin{tabular}{|c|c|c|c|c|c|c|c|c|c|c|c|c|c|c|c|c|}
\hline \multirow{3}{*}{$\begin{array}{l}\text { Core, section, } \\
\text { interval }(\mathrm{cm})\end{array}$} & \multirow{3}{*}{$\begin{array}{l}\text { Depth } \\
\text { (mbsf) }\end{array}$} & \multirow{2}{*}{$\begin{array}{l}\text { Lith. } \\
\text { unit }\end{array}$} & \multicolumn{4}{|c|}{ X-ray diffraction peak area (total counts) } & \multirow{2}{*}{$\begin{array}{l}3.54 \AA / / \\
3.58 \AA\end{array}$} & \multirow{2}{*}{\multicolumn{5}{|c|}{ Relative percent (Biscay factors) }} & \multicolumn{4}{|c|}{ Illite/smectite mixed-layer clay } \\
\hline & & & \multirow{2}{*}{$\begin{array}{c}\text { Smectite } \\
(001)\end{array}$} & \multirow{2}{*}{$\begin{array}{l}\text { Illite } \\
(001)\end{array}$} & \multirow{2}{*}{$\begin{array}{l}\text { Chlorite (002) + } \\
\text { Kaolinite (001) }\end{array}$} & \multirow{2}{*}{$\mathrm{I} / \mathrm{V}$} & & & & & & & \multirow{2}{*}{$\begin{array}{l}\mathrm{S}(001) \\
\text { saddle }\end{array}$} & $\begin{array}{l}\mathrm{S}(001) \\
\text { peak }\end{array}$ & $\begin{array}{l}\text { Saddle/ } \\
\text { Peak }\end{array}$ & \multirow{2}{*}{$\begin{array}{l}\text { Percent } \\
\text { expand }\end{array}$} \\
\hline & & & & & & & & & & & & & & & & \\
\hline 311-U1325B- & & & & & & & & & & & & & & & & \\
\hline $1 \mathrm{H}-2,43-45$ & 1.93 & IA & 145,618 & 68,651 & 144,777 & & 2.20 & 21 & 39 & 28 & 13 & & 4,651 & 7,751 & 0.60 & 66 \\
\hline $2 \mathrm{H}-5,75-77$ & 16.05 & IA & 259,790 & 61,549 & 206,636 & 8,981 & 2.09 & 28 & 27 & 30 & 14 & 1 & 5,659 & 10,516 & 0.54 & 69 \\
\hline $4 \mathrm{H}-1,75-77$ & 24.75 & $\mathrm{IB}$ & 55,971 & 123,223 & 140,234 & & 2.37 & 7 & 59 & 24 & 10 & & 2,994 & 4,100 & 0.73 & 62 \\
\hline $4 \mathrm{H}-6,75-77$ & 32.07 & IB & 90,174 & 75,099 & 118,772 & & 2.09 & 14 & 48 & 26 & 12 & & 3,176 & 4,817 & 0.66 & 65 \\
\hline $5 \mathrm{H}-4,75-77$ & 38.67 & IB & 26,435 & 123,675 & 116,345 & & 4.98 & 4 & 66 & 26 & 5 & & 2,557 & 3,017 & 0.85 & 56 \\
\hline $6 \mathrm{H}-3,75-77$ & 46.75 & $\mathrm{IB}$ & 76,487 & 58,946 & 154,994 & & 1.54 & 12 & 38 & 30 & 20 & & 4,549 & 6,161 & 0.74 & 61 \\
\hline $7 \mathrm{H}-1,58-60$ & 53.08 & ॥ & 73,121 & 56,736 & 243,164 & 10,182 & 2.39 & 9 & 28 & 43 & 18 & 1 & 3,537 & 5,067 & 0.70 & 63 \\
\hline $7 \mathrm{H}-6,75-77$ & 60.75 & ॥ & 137,471 & 62,665 & 265,222 & 5,469 & 2.14 & 15 & 27 & 39 & 18 & 1 & 5,494 & 8,730 & 0.63 & 67 \\
\hline $8 \mathrm{H}-4,75-77$ & 67.01 & \| & 168,728 & 96,435 & 258,315 & 19,326 & 2.09 & 15 & 35 & 32 & 15 & 2 & 5,619 & 8,686 & 0.65 & 66 \\
\hline $10 X-1,75-77$ & 73.55 & ॥ & 53,627 & 163,935 & 163,436 & & 3.10 & 5 & 63 & 24 & 8 & & 3,282 & 4,333 & 0.76 & 60 \\
\hline $10 \times-6,75-77$ & 80.85 & ॥ & 75,027 & 75,031 & 185,627 & 17,701 & 3.15 & 10 & 39 & 37 & 12 & 2 & 3,572 & 5,096 & 0.70 & 63 \\
\hline $12 X-3,75-77$ & 88.15 & ॥ & 135,340 & 81,607 & 208,182 & & 2.51 & 15 & 37 & 34 & 13 & & 3,828 & 6,156 & 0.62 & 67 \\
\hline $14 X-1,75-77$ & 103.05 & III & 237,201 & 105,307 & 153,204 & & 1.75 & 25 & 44 & 20 & 12 & & 5,482 & 10,338 & 0.53 & 69 \\
\hline $15 X-1,75-77$ & 112.45 & III & 157,260 & 90,853 & 272,221 & & 2.83 & 15 & 34 & 38 & 13 & & 5,368 & 9,532 & 0.56 & 68 \\
\hline $16 \mathrm{X}-6,28-30$ & 126.93 & III & 99,152 & 64,848 & 114,464 & 7,431 & 1.95 & 17 & 44 & 25 & 13 & 1 & 4,350 & 5,614 & 0.77 & 60 \\
\hline $16 \mathrm{X}-6,75-77$ & 127.40 & III & 144,289 & 113,010 & 209,551 & & 2.39 & 14 & 45 & 29 & 12 & & 5,579 & 8,148 & 0.68 & 64 \\
\hline $18 \mathrm{X}-1,75-77$ & 131.65 & III & 20,869 & 44,519 & 103,725 & 19,723 & 2.64 & 5 & 42 & 35 & 13 & 5 & 3,255 & 3,699 & 0.88 & 52 \\
\hline $19 \mathrm{X}-1,75-77$ & 141.25 & III & 172,946 & 63,844 & 140,501 & & 2.04 & 24 & 36 & 27 & 13 & & 4,522 & 7,412 & 0.61 & 67 \\
\hline $20 \times-1,5-7$ & 150.95 & III & 75,318 & 72,387 & 170,879 & 12,937 & 2.06 & 10 & 40 & 32 & 16 & 2 & 3,404 & 4,802 & 0.71 & 63 \\
\hline $20 \times-6,77-79$ & 157.61 & III & 86,649 & 76,706 & 173,265 & & 2.58 & 12 & 41 & 34 & 13 & & 3,569 & 5,353 & 0.67 & 65 \\
\hline $24 \mathrm{X}-1,75-77$ & 179.75 & III & 87,315 & 97,761 & 189,630 & & 2.83 & 10 & 46 & 33 & 12 & & 3,440 & 4,989 & 0.69 & 64 \\
\hline $24 X-5,75-77$ & 185.02 & III & 106,901 & 73,121 & 148,334 & & 2.19 & 15 & 42 & 29 & 13 & & 3,477 & 5,283 & 0.66 & 65 \\
\hline 311-U1325C- & & & & & & & & & & & & & & & & \\
\hline $1 X-2,75-77$ & 191.05 & III & 106,569 & 106,759 & 140,831 & & 2.00 & 13 & 52 & 23 & 12 & & 4,501 & 5,915 & 0.76 & 60 \\
\hline $4 \mathrm{X}-2,75-77$ & 209.99 & IV & 60,455 & 103,162 & 213,606 & & 2.52 & 7 & 46 & 34 & 13 & & 3,348 & 4,392 & 0.76 & 60 \\
\hline $6 \mathrm{X}-3,75-77$ & 221.14 & IV & 74,045 & 69,798 & 235,279 & 19,858 & 2.48 & 9 & 33 & 40 & 16 & 2 & 3,512 & 4,641 & 0.76 & 60 \\
\hline $6 X-4,75-77$ & 222.26 & IV & 34,797 & 72,240 & 274,089 & & 2.20 & 4 & 33 & 43 & 20 & & 2,899 & 3,589 & 0.81 & 57 \\
\hline $7 X-2,75-77$ & 229.45 & IV & 96,843 & 108,868 & 228,907 & & 2.65 & 10 & 44 & 34 & 13 & & 3,838 & 5,218 & 0.74 & 61 \\
\hline $8 X-1,75-77$ & 237.55 & IV & 76,429 & 127,086 & 189,819 & & 2.98 & 8 & 53 & 29 & 10 & & 3,492 & 4,837 & 0.72 & 62 \\
\hline $8 X-6,75-77$ & 244.05 & IV & 181,352 & 129,476 & 242,878 & & 2.16 & 15 & 44 & 28 & 13 & & 5,740 & 8,370 & 0.69 & 64 \\
\hline $9 X-4,75-77$ & 251.75 & IV & 64,691 & 61,926 & 198,584 & 22,291 & 2.26 & 9 & 34 & 38 & 17 & 3 & 3,305 & 4,174 & 0.79 & 58 \\
\hline $11 X-1,75-77$ & 257.85 & IV & 69,961 & 81,525 & 233,310 & 30,489 & 2.37 & 8 & 37 & 37 & 16 & 3 & 3,688 & 4,716 & 0.78 & 59 \\
\hline $11 X-2,75-77$ & 259.35 & IV & 97,596 & 104,680 & 243,781 & & 2.50 & 10 & 42 & 35 & 14 & & 3,713 & 5,218 & 0.71 & 63 \\
\hline $12 \mathrm{X}-1,75-77$ & 266.55 & IV & 72,012 & 88,193 & 186,824 & & 2.97 & 9 & 44 & 35 & 12 & & 3,492 & 4,495 & 0.78 & 59 \\
\hline $12 X-5,75-77$ & 272.42 & IV & 30,027 & 92,483 & 137,874 & & 3.96 & 4 & 55 & 33 & 8 & & 2,649 & 3,041 & 0.87 & 52 \\
\hline $15 X-1,75-77$ & 295.45 & IV & 40,910 & 69,324 & 168,694 & 25,469 & 4.41 & 6 & 41 & 40 & 9 & 4 & 2,980 & 3,551 & 0.84 & 55 \\
\hline 311-U1326C- & & & & & & & & & & & & & & & & \\
\hline $2 \mathrm{H}-2,51-53$ & 5.91 & I & 164,116 & 86,373 & 183,646 & & 2.36 & 19 & 39 & 29 & 12 & & 3,919 & 6,898 & 0.57 & 66 \\
\hline $2 \mathrm{H}-7,47-49$ & 13.37 & I & 184,947 & 96,773 & 182,747 & & 2.36 & 20 & 41 & 27 & 12 & & 3,569 & 7,147 & 0.50 & 71 \\
\hline $3 \mathrm{H}-5,80-82$ & 20.20 & 1 & 130,349 & 83,557 & 146,273 & & 2.35 & 17 & 44 & 27 & 12 & & 3,658 & 5,930 & 0.62 & 67 \\
\hline $4 \mathrm{H}-3,75-77$ & 26.65 & ॥ & 246,542 & 46,491 & 217,004 & 4,827 & 1.96 & 28 & 21 & 33 & 17 & 1 & 3,827 & 9,261 & 0.41 & 76 \\
\hline $5 X-3,75-77$ & 34.07 & ॥ & 181,932 & 75,499 & 214,827 & & 1.97 & 20 & 33 & 31 & 16 & & 3,783 & 7,435 & 0.51 & 70 \\
\hline $6 \mathrm{X}-1,75-77$ & 40.75 & ॥ & 229,250 & 52,450 & 254,623 & & 1.88 & 24 & 22 & 35 & 19 & & 3,676 & 8,592 & 0.43 & 75 \\
\hline $7 X-2,75-77$ & 51.78 & \| & 366,452 & 55,535 & 248,878 & & 1.94 & 34 & 20 & 30 & 16 & & 4,027 & 12,455 & 0.32 & 82 \\
\hline $8 X-1,75-77$ & 60.15 & ॥ & 274,234 & 48,620 & 211,380 & & 2.21 & 31 & 22 & 33 & 15 & & 4,196 & 10,240 & 0.41 & 76 \\
\hline $9 X-2,75-77$ & 71.35 & II & 294,628 & 67,815 & 258,571 & & 2.43 & 27 & 25 & 34 & 14 & & 4,094 & 10,674 & 0.38 & 78 \\
\hline $10 \mathrm{X}-1,75-77$ & 79.45 & ॥ & 100,530 & 95,677 & 194,758 & & 2.73 & 12 & 44 & 33 & 12 & & 3,393 & 5,240 & 0.65 & 66 \\
\hline
\end{tabular}


Table T1 (continued). (Continued on next page.)

\begin{tabular}{|c|c|c|c|c|c|c|c|c|c|c|c|c|c|c|c|c|}
\hline \multirow[b]{2}{*}{$\begin{array}{l}\text { Core, section, } \\
\text { interval }(\mathrm{cm})\end{array}$} & \multirow[b]{2}{*}{$\begin{array}{l}\text { Depth } \\
\text { (mbsf) }\end{array}$} & \multirow[b]{2}{*}{$\begin{array}{l}\text { Lith. } \\
\text { unit }\end{array}$} & \multicolumn{4}{|c|}{ X-ray diffraction peak area (total counts) } & \multirow[b]{2}{*}{$\begin{array}{l}3.54 \AA / \\
3.58 \AA\end{array}$} & \multirow{2}{*}{\multicolumn{5}{|c|}{ Relative percent (Biscay factors) }} & \multicolumn{4}{|c|}{ Illite/smectite mixed-layer clay } \\
\hline & & & $\begin{array}{c}\text { Smectite } \\
(001)\end{array}$ & $\begin{array}{l}\text { Illite } \\
(001)\end{array}$ & $\begin{array}{l}\text { Chlorite }(002)+ \\
\text { Kaolinite }(001)\end{array}$ & $\mathrm{I} / \mathrm{V}$ & & & & & & & $\begin{array}{l}\mathrm{S}(001) \\
\text { saddle }\end{array}$ & $\begin{array}{l}\mathrm{S}(001) \\
\text { peak }\end{array}$ & $\begin{array}{l}\text { Saddle/ } \\
\text { Peak }\end{array}$ & $\begin{array}{l}\text { Percent } \\
\text { expand }\end{array}$ \\
\hline 311-U1326D- & & & & & & & & & & & & & & & & \\
\hline $2 X-1,72-74$ & 89.12 & ॥ & 362,230 & 36,113 & 176,236 & & 2.37 & 42 & 17 & 29 & 12 & & 3,830 & 12,335 & 0.31 & 82 \\
\hline $2 X-5,75-77$ & 95.15 & II & 181,800 & 59,203 & 176,097 & & 2.17 & 24 & 31 & 31 & 14 & & 4,039 & 7,310 & 0.55 & 68 \\
\hline $3 X-3,75-77$ & 101.85 & ॥ & 275,328 & 38,389 & 208,017 & & 0.88 & 33 & 18 & 23 & 26 & & 3,997 & 9,647 & 0.41 & 76 \\
\hline $4 X-4,75-77$ & 112.51 & ॥ & 234,784 & 55,157 & 180,762 & & 2.59 & 29 & 27 & 32 & 12 & & 3,873 & 9,036 & 0.43 & 75 \\
\hline $5 X-1,75-77$ & 118.05 & ॥ & 123,316 & 78,569 & 231,166 & & 2.05 & 14 & 35 & 35 & 17 & & 3,499 & 6,054 & 0.58 & 67 \\
\hline $5 X-6,75-77$ & 124.35 & ॥ & 112,230 & 107,751 & 258,580 & & 2.41 & 11 & 41 & 34 & 14 & & 3,499 & 5,534 & 0.63 & 67 \\
\hline $6 \mathrm{X}-6,75-77$ & 134.20 & ॥ & 65,793 & 91,892 & 211,449 & & 2.49 & 8 & 43 & 35 & 14 & & 3,142 & 4,390 & 0.72 & 62 \\
\hline $7 X-4,75-77$ & 140.94 & ॥ & 41,605 & 86,350 & 192,410 & & 2.46 & 5 & 45 & 35 & 14 & & 2,877 & 3,644 & 0.79 & 58 \\
\hline $8 X-3,75-77$ & 149.51 & III & 13,466 & 83,547 & 155,369 & 58,382 & 3.55 & 2 & 47 & 34 & 10 & 8 & 2,507 & 2,746 & 0.91 & 49 \\
\hline $9 X-1,75-77$ & 156.65 & III & 89,771 & 35,631 & 120,886 & & 2.27 & 19 & 30 & 35 & 16 & & 3,589 & 5,513 & 0.65 & 66 \\
\hline $10 \times-1,75-77$ & 166.35 & III & 92,678 & 58,173 & 168,618 & & 2.37 & 14 & 35 & 36 & 15 & & 3,629 & 5,378 & 0.67 & 65 \\
\hline $10 X-4,75-77$ & 170.77 & III & 75,167 & 48,856 & 124,775 & & 2.59 & 14 & 38 & 35 & 13 & & 3,092 & 4,364 & 0.71 & 63 \\
\hline $11 X-4,75-77$ & 179.44 & III & 19,508 & 44,792 & 253,182 & 7,808 & 2.24 & 3 & 25 & 49 & 22 & 1 & 2,405 & 2,820 & 0.85 & 56 \\
\hline $12 X-2,75-77$ & 186.80 & III & 48,686 & 98,675 & 169,288 & & 2.69 & 6 & 50 & 32 & 12 & & 3,106 & 4,032 & 0.77 & 60 \\
\hline $13 X-1,75-77$ & 195.15 & III & 14,861 & 53,520 & 233,263 & 6,955 & 2.49 & 2 & 30 & 47 & 19 & 1 & 2,575 & 2,887 & 0.89 & 51 \\
\hline $14 X-1,75-77$ & 204.75 & III & 39,956 & 94,068 & 202,596 & 22,906 & 3.01 & 5 & 45 & 36 & 12 & 3 & 3,162 & 3,991 & 0.79 & 58 \\
\hline $15 X-1,73-75$ & 214.33 & III & 41,741 & 109,397 & 202,723 & & 3.12 & 5 & 49 & 35 & 11 & & 2,933 & 3,863 & 0.76 & 60 \\
\hline $16 \mathrm{X}-1,75-77$ & 224.05 & III & 71,390 & 109,103 & 218,355 & & 2.64 & 8 & 46 & 34 & 13 & & 3,423 & 4,619 & 0.74 & 61 \\
\hline $17 X-1,75-77$ & 233.65 & III & 25,527 & 90,374 & 154,120 & & 2.38 & 4 & 52 & 31 & 13 & & 2,981 & 3,449 & 0.86 & 54 \\
\hline $17 X-3,0-100$ & 235.90 & III & 58,815 & 53,093 & 170,389 & 23,825 & 2.65 & 9 & 33 & 39 & 15 & 4 & 3,618 & 4,664 & 0.78 & 59 \\
\hline $17 X-5,75-77$ & 239.65 & III & 25,490 & 58,856 & 244,420 & 10,365 & 3.02 & 3 & 31 & 48 & 16 & 1 & 3,133 & 3,599 & 0.87 & 52 \\
\hline $20 \times-3,75-77$ & 264.50 & III & 105,108 & 101,827 & 192,621 & & 2.57 & 12 & 45 & 31 & 12 & & 3,974 & 5,592 & 0.71 & 63 \\
\hline $20 \times-7,74-76$ & 269.78 & III & 39,121 & 80,237 & 154,762 & & 2.52 & 6 & 48 & 33 & 13 & & 3,011 & 3,596 & 0.84 & 55 \\
\hline 311-U1327C- & & & & & & & & & & & & & & & & \\
\hline $1 \mathrm{H}-2,75-77$ & 2.25 & I & 256,280 & 76,041 & 219,424 & 10,687 & 2.40 & 25 & 30 & 31 & 13 & 1 & 5,375 & 10,310 & 0.52 & 69 \\
\hline $2 \mathrm{H}-2,75-77$ & 8.35 & 1 & 150,519 & 73,697 & 182,232 & 8,003 & 2.60 & 18 & 36 & 32 & 12 & 1 & 7,392 & 8,892 & 0.83 & 56 \\
\hline $3 \mathrm{H}-1,75-77$ & 16.35 & 1 & 102,230 & 72,625 & 144,826 & 7,418 & 2.10 & 15 & 42 & 28 & 14 & 1 & 7,114 & 7,714 & 0.92 & 49 \\
\hline $3 \mathrm{H}-5,75-77$ & 22.35 & I & 40,443 & 91,460 & 169,793 & 27,361 & 2.36 & 5 & 47 & 31 & 13 & 4 & 5,861 & 6,012 & 0.97 & 46 \\
\hline $4 \mathrm{H}-3,75-77$ & 28.85 & I & 125,152 & 56,550 & 156,475 & 4,702 & 1.95 & 19 & 34 & 31 & 16 & 1 & 6,580 & 8,143 & 0.81 & 57 \\
\hline $5 \mathrm{H}-3,75-77$ & 38.35 & I & 101,917 & 70,061 & 234,295 & 17,652 & 3.20 & 12 & 32 & 41 & 13 & 2 & 7,061 & 7,818 & 0.90 & 50 \\
\hline $7 \mathrm{H}-2,75-77$ & 48.35 & I & 170,263 & 70,703 & 187,573 & & 2.07 & 21 & 34 & 31 & 15 & & 7,747 & 10,047 & 0.77 & 60 \\
\hline $8 \mathrm{H}-1,75-77$ & 56.35 & 1 & 268,308 & 63,666 & 226,219 & & 2.07 & 28 & 26 & 31 & 15 & & 7,810 & 12,496 & 0.63 & 66 \\
\hline $9 \mathrm{H}-1,75-77$ & 65.85 & I & 97,992 & 94,034 & 202,779 & 21,951 & 2.55 & 11 & 42 & 32 & 13 & 2 & 7,470 & 7,980 & 0.94 & 47 \\
\hline $9 \mathrm{H}-6,75-77$ & 73.35 & I & 46,766 & 49,138 & 119,787 & 18,302 & 2.60 & 9 & 39 & 35 & 13 & 4 & 6,667 & 6,979 & 0.96 & 45 \\
\hline $10 \mathrm{H}-1,96-98$ & 75.56 & I & 157,834 & 68,649 & 193,434 & & 1.94 & 19 & 34 & 31 & 16 & & 4,085 & 6,668 & 0.61 & 67 \\
\hline $10 \mathrm{H}-6,75-77$ & 81.50 & 1 & 135,678 & 80,194 & 233,227 & 18,679 & 2.35 & 14 & 34 & 35 & 15 & 2 & 7,787 & 9,011 & 0.86 & 52 \\
\hline $11 \mathrm{H}-4,75-77$ & 89.35 & 1 & 53,315 & 72,592 & 172,086 & 20,533 & 2.22 & 8 & 41 & 34 & 15 & 3 & 6,421 & 6,686 & 0.96 & 45 \\
\hline $12 X-6,75-77$ & 99.87 & ॥ & 238,076 & 95,343 & 171,022 & & 1.90 & 25 & 40 & 23 & 12 & & 5,285 & 9,599 & 0.55 & 69 \\
\hline $13 X-4,75-77$ & 107.75 & ॥ & 62,793 & 68,972 & 156,312 & 16,894 & 2.66 & 9 & 41 & 34 & 13 & 3 & 6,584 & 7,043 & 0.93 & 48 \\
\hline $14 X-3,75-77$ & 115.85 & ॥ & 71,740 & 109,004 & 215,572 & & 2.55 & 8 & 46 & 33 & 13 & & 6,371 & 7,037 & 0.91 & 49 \\
\hline $16 \times-1,75-77$ & 124.55 & ॥ & 56,280 & 58,947 & 144,569 & 10,518 & 2.29 & 10 & 40 & 34 & 15 & 2 & 7,043 & 7,433 & 0.95 & 46 \\
\hline $16 \mathrm{X}-3,75-77$ & 127.45 & ॥ & 109,329 & 69,252 & 217,386 & 10,097 & 2.36 & 13 & 33 & 37 & 16 & 1 & 6,125 & 7,714 & 0.79 & 58 \\
\hline $18 X-1,72-74$ & 142.22 & ॥ & 144,492 & 40,693 & 84,663 & 2,764 & 2.64 & 30 & 34 & 26 & 10 & 1 & 7,493 & 8,829 & 0.85 & 56 \\
\hline $18 \times-2,0-5$ & 142.35 & ॥ & 118,421 & 33,198 & 75,488 & & 2.44 & 29 & 33 & 27 & 11 & & 4,166 & 5,711 & 0.73 & 62 \\
\hline $18 \mathrm{X}-2,130-150$ & 143.65 & $\|$ & 101,190 & 40,889 & 101,571 & & 2.01 & 22 & 35 & 29 & 14 & & 3,940 & 5,326 & 0.74 & 61 \\
\hline $19 X-1,75-77$ & 151.85 & II & 98,030 & 39,034 & 105,135 & & 2.11 & 21 & 34 & 31 & 15 & & 6,673 & 7,573 & 0.88 & 52 \\
\hline $19 X-6,75-77$ & 158.35 & II & 118,905 & 51,388 & 104,528 & & 2.21 & 22 & 39 & 27 & 12 & & 7,442 & 8,275 & 0.90 & 50 \\
\hline $20 \times-3,75-77$ & 163.83 & $\|$ & 167,713 & 70,924 & 178,998 & & 1.79 & 21 & 35 & 28 & 16 & & 4,577 & 7,278 & 0.63 & 66 \\
\hline
\end{tabular}


Table T1 (continued). (Continued on next page.)

\begin{tabular}{|c|c|c|c|c|c|c|c|c|c|c|c|c|c|c|c|c|}
\hline \multirow{2}{*}{$\begin{array}{l}\text { Core, section, } \\
\text { interval }(\mathrm{cm})\end{array}$} & \multirow{2}{*}{$\begin{array}{l}\text { Depth } \\
\text { (mbsf) }\end{array}$} & \multirow[b]{2}{*}{$\begin{array}{l}\text { Lith. } \\
\text { unit }\end{array}$} & \multicolumn{4}{|c|}{ X-ray diffraction peak area (total counts) } & \multirow{2}{*}{$\begin{array}{l}3.54 \AA \AA \\
3.58 \AA\end{array}$} & \multirow{2}{*}{\multicolumn{5}{|c|}{ Relative percent (Biscay factors) }} & \multicolumn{4}{|c|}{ Illite/smectite mixed-layer clay } \\
\hline & & & $\begin{array}{l}\text { Smectite } \\
(001)\end{array}$ & $\begin{array}{l}\text { Illite } \\
(001)\end{array}$ & $\begin{array}{l}\text { Chlorite }(002)+ \\
\text { Kaolinite }(001)\end{array}$ & $\mathrm{I} / \mathrm{V}$ & & & & & & & $\begin{array}{l}\mathrm{S}(001) \\
\text { saddle }\end{array}$ & $\begin{array}{l}S(001) \\
\text { peak }\end{array}$ & $\begin{array}{l}\text { Saddle/ } \\
\text { Peak }\end{array}$ & $\begin{array}{l}\text { Percent } \\
\text { expand }\end{array}$ \\
\hline $21 X-1,75-77$ & 171.15 & III & 97,114 & 89,810 & 189,757 & & 2.42 & 12 & 43 & 32 & 13 & & 3,555 & 5,298 & 0.67 & 65 \\
\hline $21 X-6,75-77$ & 178.17 & IIII & 62,099 & 55,319 & 103,942 & & 2.61 & 13 & 45 & 31 & 12 & & 6,666 & 7,178 & 0.93 & 48 \\
\hline $21 X-7,0-1$ & 178.43 & III & 83,827 & 78,057 & 107,581 & & 2.62 & 14 & 51 & 25 & 10 & & 4,181 & 5,244 & 0.80 & 58 \\
\hline $22 X-5,75-77$ & 186.00 & III & 44,466 & 68,416 & 185,137 & 15,499 & 2.73 & 6 & 39 & 39 & 14 & 2 & 2,998 & 3,788 & 0.79 & 58 \\
\hline $25 X-2,77-79$ & 200.88 & IIII & 43,317 & 84,157 & 151,989 & & 2.50 & 6 & 49 & 32 & 13 & & 2,883 & 3,335 & 0.86 & 53 \\
\hline $26 X-1,75-77$ & 209.65 & III & 59,274 & 161,121 & 225,754 & & 4.02 & 5 & 56 & 31 & 8 & & 3,522 & 4,442 & 0.79 & 58 \\
\hline $27 X-1,75-77$ & 219.25 & III & 67,246 & 90,811 & 161,406 & & 3.20 & 9 & 48 & 33 & 10 & & 6,642 & 7,314 & 0.91 & 49 \\
\hline $27 X-5,75-77$ & 224.88 & IIII & 298,684 & 76,204 & 167,537 & & 2.12 & 32 & 32 & 24 & 11 & & 4,798 & 10,453 & 0.46 & 73 \\
\hline $28 X-2,74-76$ & 230.34 & III & 97,648 & 87,679 & 205,050 & & 2.31 & 11 & 41 & 33 & 14 & & 3,463 & 4,988 & 0.69 & 64 \\
\hline $29 X-1,75-77$ & 238.45 & IIII & 46,829 & 92,360 & 106,892 & & 3.11 & 7 & 59 & 26 & 8 & & 2,643 & 3,435 & 0.77 & 60 \\
\hline $29 X-5,75-77$ & 244.30 & IIII & 55,605 & 73,855 & 139,283 & 21,377 & 2.94 & 9 & 45 & 32 & 11 & 3 & 3,461 & 4,258 & 0.81 & 57 \\
\hline $30 X-5,70-72$ & 253.31 & IIII & 109,979 & 144,143 & 282,440 & & 3.10 & 9 & 46 & 34 & 11 & & 4,157 & 6,008 & 0.69 & 64 \\
\hline $31 X-5,75-77$ & 263.81 & IIII & 109,335 & 24,891 & 60,971 & & 2.11 & 33 & 30 & 25 & 12 & & 6,776 & 7,318 & 0.93 & 48 \\
\hline $32 X-2,75-77$ & 268.95 & IIII & 69,442 & 140,233 & 177,811 & & 3.11 & 7 & 57 & 27 & 9 & & 3,094 & 4,213 & 0.73 & 62 \\
\hline $33 X-2,75-77$ & 278.55 & IIII & 53,202 & 109,003 & 95,289 & & 2.62 & 8 & 64 & 20 & 8 & & 3,192 & 3,867 & 0.83 & 56 \\
\hline $34 X-2,77-79$ & 288.17 & III & 92,256 & 125,531 & 137,241 & & 3.47 & 11 & 58 & 25 & 7 & & 3,715 & 5,365 & 0.69 & 64 \\
\hline $35 X-1,75-77$ & 296.25 & III & 107,844 & 45,510 & 95,624 & & 3.20 & 22 & 38 & 30 & 9 & & 3,659 & 5,380 & 0.68 & 64 \\
\hline \multicolumn{17}{|l|}{ 311-U1327D- } \\
\hline $5 X-1,65-75$ & 126.95 & II & 45,362 & 93,425 & 128,511 & & 2.38 & 7 & 55 & 27 & 11 & & 2,893 & 3,620 & 0.80 & 58 \\
\hline $11 X-1,0-8$ & 157.10 & II & 20,965 & 54,134 & 50,421 & & 2.69 & 6 & 64 & 22 & 8 & & 2,312 & 2,709 & 0.85 & 56 \\
\hline 311-U1328B- & & & & & & & & & & & & & & & & \\
\hline $1 \mathrm{H}-1,71-73$ & 0.71 & 1 & 23,999 & 23,958 & 34,872 & & 2.23 & 13 & 51 & 25 & 11 & & 963 & 1,394 & 0.69 & 64 \\
\hline $1 \mathrm{H}-3,60-62$ & 3.60 & 1 & 38,356 & 36,976 & 34,052 & & 2.07 & 15 & 58 & 18 & 9 & & 1,084 & 1,768 & 0.61 & 67 \\
\hline $4 \mathrm{P}-1,0-11$ & 14.50 & 1 & 88,812 & 29,316 & 44,333 & & 1.54 & 30 & 40 & 18 & 12 & & 1,751 & 3,303 & 0.53 & 69 \\
\hline $5 \mathrm{H}-1,68-70$ & 17.18 & 1 & 15,671 & 30,550 & 52,685 & & 2.37 & 6 & 50 & 30 & 13 & & 965 & 1,228 & 0.79 & 58 \\
\hline $6 \mathrm{X}-1,0-20$ & 18.40 & 1 & 74,851 & 22,912 & 50,147 & & 1.59 & 28 & 34 & 23 & 15 & & 1,545 & 2,920 & 0.53 & 69 \\
\hline $6 X-1,36-38$ & 18.76 & 1 & 29,223 & 20,304 & 51,742 & & 2.42 & 14 & 38 & 34 & 14 & & 1,093 & 1,611 & 0.68 & 64 \\
\hline $8 \mathrm{H}-1,75-77$ & 28.75 & 1 & 21,903 & 44,514 & 60,169 & & 2.44 & 7 & 56 & 27 & 11 & & 1,159 & 1,594 & 0.73 & 62 \\
\hline $9 \mathrm{H}-1,75-77$ & 38.25 & 1 & 26,444 & 27,352 & 41,728 & & 1.93 & 12 & 50 & 25 & 13 & & 1,021 & 1,519 & 0.67 & 65 \\
\hline $9 \mathrm{H}-6,75-77$ & 45.56 & 1 & 26,421 & 18,444 & 44,493 & & 2.09 & 14 & 39 & 32 & 15 & & 1,022 & 1,556 & 0.66 & 65 \\
\hline $10 \mathrm{H}-5,75-77$ & 53.75 & 1 & 28,584 & 20,727 & 55,875 & & 2.71 & 13 & 37 & 37 & 13 & & 1,053 & 1,580 & 0.67 & 65 \\
\hline 311-U1328C- & & & & & & & & & & & & & & & & \\
\hline $1 \mathrm{H}-4,75-77$ & 61.68 & 1 & 21,064 & 13,535 & 45,013 & 3,247 & 2.47 & 13 & 32 & 38 & 15 & 2 & 1,010 & 1,442 & 0.70 & 63 \\
\hline $2 \mathrm{H}-5,75-77$ & 72.75 & 1 & 13,002 & 13,258 & 26,838 & 2,573 & 2.58 & 11 & 43 & 32 & 12 & 2 & 927 & 1,114 & 0.83 & 56 \\
\hline $3 \mathrm{H}-6,76-78$ & 83.76 & 1 & 32,634 & 22,948 & 54,651 & & 2.23 & 14 & 39 & 32 & 14 & & 1,153 & 1,701 & 0.68 & 64 \\
\hline $6 \mathrm{H}-1,75-77$ & 94.75 & 1 & 15,956 & 23,826 & 55,491 & 3,194 & 2.23 & 7 & 42 & 34 & 15 & 1 & 984 & 1,293 & 0.76 & 60 \\
\hline $6 \mathrm{H}-6,75-77$ & 99.75 & 1 & 61,107 & 15,442 & 33,451 & & 2.22 & 32 & 33 & 24 & 11 & & 1,219 & 2,320 & 0.53 & 69 \\
\hline $6 \mathrm{H}-8,75-77$ & 102.14 & 1 & 60,680 & 23,373 & 39,939 & & 1.69 & 26 & 40 & 21 & 13 & & 1,139 & 2,370 & 0.48 & 72 \\
\hline $7 X-1,73-75$ & 104.23 & 1 & 34,786 & 18,855 & 23,735 & & 2.02 & 22 & 48 & 20 & 10 & & 1,055 & 1,674 & 0.63 & 67 \\
\hline $7 X-4,75-77$ & 108.65 & 1 & 59,885 & 7,647 & 46,093 & & 2.91 & 33 & 17 & 38 & 13 & & 1,120 & 2,538 & 0.44 & 74 \\
\hline $8 X-2,74-76$ & 112.54 & 1 & 50,878 & 14,529 & 52,789 & 1,012 & 3.56 & 24 & 27 & 38 & 11 & 1 & 1,156 & 2,211 & 0.52 & 69 \\
\hline $9 X-1,78-80$ & 120.68 & 1 & 48,701 & 8,043 & 37,589 & 1,504 & 1.89 & 31 & 20 & 31 & 16 & 1 & 1,077 & 2,114 & 0.51 & 70 \\
\hline $9 X-4,72-74$ & 124.62 & 1 & 71,345 & 14,832 & 49,130 & & 2.56 & 31 & 26 & 31 & 12 & & 1,071 & 2,482 & 0.43 & 75 \\
\hline $9 X-5,78-80$ & 126.18 & 1 & 94,370 & 13,061 & 52,412 & & 3.42 & 38 & 21 & 32 & 9 & & 1,271 & 3,404 & 0.37 & 79 \\
\hline $10 X-1,75-77$ & 130.35 & 1 & 49,817 & 16,905 & 40,313 & & 2.17 & 25 & 34 & 28 & 13 & & 1,158 & 2,087 & 0.55 & 71 \\
\hline $10 X-3,75-77$ & 133.35 & II & 11,847 & 33,677 & 24,573 & & 3.59 & 6 & 69 & 20 & 5 & & 845 & 1,041 & 0.81 & 57 \\
\hline $11 X-1,75-77$ & 140.05 & II & 32,020 & 21,293 & 27,659 & & 2.42 & 19 & 49 & 23 & 9 & & 1,131 & 1,659 & 0.68 & 64 \\
\hline $11 X-2,75-77$ & 141.55 & II & 25,981 & 14,257 & 23,009 & & 2.93 & 20 & 44 & 27 & 9 & & 1,051 & 1,452 & 0.72 & 62 \\
\hline
\end{tabular}


Table T1 (continued). (Continued on next page.)

\begin{tabular}{|c|c|c|c|c|c|c|c|c|c|c|c|c|c|c|c|c|}
\hline \multirow[b]{2}{*}{$\begin{array}{l}\text { Core, section, } \\
\text { interval }(\mathrm{cm})\end{array}$} & \multirow[b]{2}{*}{$\begin{array}{l}\text { Depth } \\
\text { (mbsf) }\end{array}$} & \multirow[b]{2}{*}{$\begin{array}{l}\text { Lith. } \\
\text { unit }\end{array}$} & \multicolumn{4}{|c|}{ X-ray diffraction peak area (total counts) } & \multirow[b]{2}{*}{$\begin{array}{l}3.54 \AA / \\
3.58 \AA\end{array}$} & \multirow{2}{*}{\multicolumn{5}{|c|}{ Relative percent (Biscay factors) }} & \multicolumn{4}{|c|}{ Illite/smectite mixed-layer clay } \\
\hline & & & $\begin{array}{c}\text { Smectite } \\
(001)\end{array}$ & $\begin{array}{l}\text { Illite } \\
(001)\end{array}$ & $\begin{array}{c}\text { Chlorite }(002)+ \\
\text { Kaolinite }(001)\end{array}$ & $\mathrm{I} / \mathrm{V}$ & & & & & & & $\begin{array}{l}\mathrm{S}(001) \\
\text { saddle }\end{array}$ & $\begin{array}{c}\mathrm{S}(001) \\
\text { peak }\end{array}$ & $\begin{array}{l}\text { Saddle/ } \\
\text { Peak }\end{array}$ & $\begin{array}{l}\text { Percent } \\
\text { expand }\end{array}$ \\
\hline $12 \mathrm{X}-1,75-77$ & 149.65 & $\|$ & 15,223 & 18,756 & 36,735 & 2,662 & 2.89 & 9 & 45 & 33 & 11 & 2 & 951 & 1,223 & 0.78 & 59 \\
\hline $13 X-1,75-77$ & 159.35 & II & 15,500 & 23,961 & 50,446 & & 2.49 & 7 & 45 & 34 & 14 & & 937 & 1,164 & 0.80 & 58 \\
\hline $13 X-2,75-77$ & 160.82 & ॥ & 18,391 & 16,388 & 38,601 & 5,066 & 2.26 & 11 & 39 & 32 & 14 & 3 & 962 & 1,268 & 0.76 & 60 \\
\hline $14 X-2,75-77$ & 170.28 & II & 4,962 & 17,628 & 38,189 & 8,482 & 2.46 & 3 & 44 & 34 & 14 & 5 & 845 & 963 & 0.88 & 52 \\
\hline $15 X-2,75-77$ & 180.15 & II & 7,260 & 16,440 & 39,341 & 3,809 & 2.76 & 5 & 42 & 37 & 13 & 2 & 833 & 989 & 0.84 & 55 \\
\hline $15 X-5,75-77$ & 184.62 & ॥ & 8,414 & 28,811 & 41,299 & & 2.79 & 4 & 56 & 29 & 11 & & 864 & 995 & 0.87 & 52 \\
\hline $16 X-5,75-77$ & 194.25 & ॥ & 13,527 & 29,640 & 40,495 & & 2.98 & 6 & 56 & 28 & 10 & & 970 & 1,225 & 0.79 & 58 \\
\hline $17 X-4,75-77$ & 202.35 & III & 15,743 & 22,033 & 41,130 & 4,059 & 2.62 & 8 & 46 & 31 & 12 & 2 & 1,001 & 1,524 & 0.66 & 65 \\
\hline $19 X-2,75-77$ & 217.78 & III & 28,705 & 20,793 & 48,814 & 5,452 & 2.74 & 13 & 39 & 33 & 12 & 3 & 1,230 & 1,746 & 0.70 & 63 \\
\hline $19 X-4,24-26$ & 220.27 & IIII & 34,560 & 16,052 & 45,978 & 1,532 & 2.54 & 18 & 33 & 34 & 13 & 1 & 1,379 & 1,938 & 0.71 & 63 \\
\hline $19 X-4,32-36$ & 220.35 & III & 48,771 & 19,476 & 47,399 & & 2.50 & 22 & 35 & 31 & 12 & & 1,480 & 2,183 & 0.68 & 64 \\
\hline $19 X-4,77-79$ & 220.80 & III & 20,084 & 30,026 & 39,573 & & 2.42 & 9 & 55 & 26 & 11 & & 1,037 & 1,439 & 0.72 & 62 \\
\hline $20 \times-3,75-77$ & 229.10 & III & 6,400 & 8,899 & 26,289 & 4,997 & 4.01 & 6 & 36 & 42 & 11 & 5 & 766 & 850 & 0.90 & 50 \\
\hline $20 \times-5,75-77$ & 231.82 & III & 12,636 & 34,695 & 42,217 & & 2.51 & 5 & 59 & 26 & 10 & & 944 & 1,667 & 0.57 & 67 \\
\hline $22 X-5,75-77$ & 251.06 & III & 9,815 & 11,507 & 30,915 & 1,379 & 2.91 & 8 & 39 & 39 & 13 & 1 & 870 & 1,037 & 0.84 & 55 \\
\hline $22 X-6,75-77$ & 252.56 & IIII & 35,738 & 38,584 & 57,542 & & 3.20 & 12 & 51 & 29 & 9 & & 1,313 & 1,966 & 0.67 & 65 \\
\hline $22 \mathrm{X}-\mathrm{CC}, 12-14$ & 253.70 & III & 16,131 & 5,888 & 27,746 & & 3.70 & 17 & 25 & 46 & 12 & & 1,025 & 1,191 & 0.86 & 54 \\
\hline $25 \mathrm{X}-1,78-80$ & 274.88 & III & 47,449 & 16,345 & 11,613 & & 2.27 & 35 & 48 & 12 & 5 & & 1,205 & 1,994 & 0.60 & 66 \\
\hline $25 X-2,77-79$ & 276.37 & III & 25,658 & 13,277 & 43,862 & & 2.04 & 15 & 32 & 35 & 17 & & 1,107 & 1,454 & 0.76 & 60 \\
\hline $26 \times-2,73-75$ & 285.93 & III & 17,508 & 22,794 & 42,050 & & 2.30 & 9 & 47 & 30 & 13 & & 921 & 1,245 & 0.74 & 61 \\
\hline $26 \mathrm{X}-4,71-73$ & 288.91 & III & 30,005 & 9,723 & 25,391 & & 2.65 & 25 & 32 & 31 & 12 & & 1,124 & 1,592 & 0.71 & 63 \\
\hline $27 X-5,75-77$ & 299.86 & III & 8,180 & 9,410 & 21,533 & 1,923 & 2.87 & 9 & 41 & 35 & 12 & 2 & 770 & 904 & 0.85 & 56 \\
\hline $\begin{array}{l}\text { 311-U1329B- } \\
1 \mathrm{H}-1,74-76\end{array}$ & 074 & 1 & 64624 & 69838 & 186089 & & & 0 & 39 & 52 & & & 3134 & 4364 & 27 & 62 \\
\hline 311-U1329C- & 0.14 & 1 & $64,0<4$ & 69,838 & 186,089 & & & 9 & 39 & 52 & & & 3,134 & 4,364 & 0.72 & 62 \\
\hline $2 \mathrm{H}-2,75-77$ & 10.35 & । & 57,732 & 81,182 & 122,863 & & & 9 & 52 & 39 & & & 2,731 & 3,724 & 0.73 & 62 \\
\hline $3 \mathrm{H}-1,77-79$ & 18.37 & i & 116,637 & 104,382 & 135,706 & & & 14 & 52 & 34 & & & 3,629 & 5,669 & 0.64 & 66 \\
\hline $3 \mathrm{H}-2,75-77$ & 19.85 & i & 76,719 & 97,657 & 178,766 & & & 9 & 47 & 43 & & & 3,373 & 4,977 & 0.68 & 64 \\
\hline $4 \mathrm{H}-1,75-77$ & 27.85 & 1 & 131,347 & 70,991 & 157,480 & 17,344 & & 18 & 38 & 42 & & 2 & 4,907 & 6,954 & 0.71 & 63 \\
\hline $4 \mathrm{H}-6,75-77$ & 35.35 & 1 & 85,700 & 67,819 & 169,943 & 8,816 & & 12 & 38 & 48 & & 1 & 4,114 & 5,653 & 0.73 & 62 \\
\hline $5 \mathrm{H}-4,74-76$ & 41.84 & ॥ & 222,962 & 46,764 & 98,343 & & & 37 & 31 & 32 & & & 5,786 & 9,231 & 0.63 & 67 \\
\hline $6 \mathrm{H}-3,75-77$ & 49.85 & II & 37,733 & 77,041 & 199,485 & & 2.47 & 5 & 41 & 38 & 15 & & 2,629 & 3,251 & 0.81 & 57 \\
\hline $8 \mathrm{H}-1,75-77$ & 58.35 & ॥ & 110,566 & 42,405 & 91,575 & & 4.20 & 24 & 37 & 32 & 8 & & 3,385 & 5,245 & 0.65 & 66 \\
\hline $8 \mathrm{H}-6,75-77$ & 65.85 & $\|$ & 46,182 & 49,413 & 177,854 & 13,950 & 2.85 & 8 & 32 & 43 & 15 & 2 & 2,835 & 3,757 & 0.75 & 61 \\
\hline $9 \mathrm{H}-4,75-77$ & 72.35 & II & 22,468 & 67,301 & 157,344 & 18,966 & 5.08 & 4 & 43 & 42 & 8 & 3 & 2,409 & 2,810 & 0.86 & 54 \\
\hline $10 \mathrm{H}-3,75-77$ & 80.35 & ॥ & 62,402 & 85,987 & 195,504 & & 3.65 & 8 & 43 & 38 & 11 & & 3,066 & 4,153 & 0.74 & 61 \\
\hline $11 \mathrm{H}-2,75-77$ & 88.35 & ॥ & 126,721 & 43,240 & 94,754 & & 3.03 & 26 & 35 & 29 & 10 & & 3,501 & 5,748 & 0.61 & 67 \\
\hline $12 \mathrm{H}-1,75-77$ & 96.35 & $\|$ & 89,971 & 47,571 & 52,866 & & 5.65 & 23 & 49 & 23 & 4 & & 2,754 & 4,428 & 0.62 & 67 \\
\hline $12 \mathrm{H}-2,75-77$ & 97.83 & ॥ & 87,409 & 143,721 & 134,903 & & 3.15 & 9 & 62 & 22 & 7 & & 3,151 & 4,873 & 0.65 & 66 \\
\hline $13 \mathrm{H}-1,75-77$ & 105.85 & ॥ & 61,366 & 77,707 & 190,995 & & 4.34 & 8 & 41 & 41 & 9 & & 3,073 & 4,207 & 0.73 & 62 \\
\hline $13 \mathrm{H}-7,72-74$ & 114.82 & II & 99,066 & 61,797 & 222,271 & & 3.45 & 13 & 31 & 44 & 13 & & 3,372 & 5,549 & 0.61 & 67 \\
\hline $15 \mathrm{H}-6,75-77$ & 124.85 & ॥ & 117,222 & 59,916 & 98,035 & 14,708 & 2.54 & 21 & 42 & 25 & 10 & 3 & 4,027 & 5,606 & 0.72 & 62 \\
\hline $16 \mathrm{H}-6,75-77$ & 134.35 & ॥ & 127,120 & 29,156 & 83,225 & & 2.27 & 31 & 28 & 28 & 12 & & 3,641 & 5,783 & 0.63 & 67 \\
\hline $18 X-1,57-59$ & 140.77 & III & 134,316 & 26,306 & 69,069 & & 2.65 & 36 & 28 & 27 & 10 & & 3,442 & 5,467 & 0.63 & 67 \\
\hline $18 X-3,57-59$ & 143.77 & III & 71,262 & 51,703 & 164,080 & 8,654 & 3.22 & 12 & 34 & 41 & 13 & 1 & 3,312 & 4,728 & 0.70 & 63 \\
\hline $19 \times-1,75-77$ & 150.65 & III & 66,437 & 48,373 & 133,423 & 14,346 & 2.26 & 12 & 36 & 34 & 15 & 3 & 3,358 & 4,255 & 0.79 & 58 \\
\hline $19 X-5,75-77$ & 156.65 & III & 173,344 & 33,014 & 84,684 & & 1.52 & 37 & 28 & 22 & 14 & & 4,191 & 6,766 & 0.62 & 67 \\
\hline $20 \mathrm{X}-1,75-77$ & 160.35 & III & 230,447 & 42,628 & 103,176 & & 2.56 & 38 & 28 & 24 & 10 & & 4,516 & 8,444 & 0.53 & 69 \\
\hline $21 \mathrm{X}-1,75-77$ & 170.05 & III & 341,626 & 40,409 & 132,606 & & 1.40 & 44 & 21 & 20 & 14 & & 4,765 & 10,596 & 0.45 & 73 \\
\hline
\end{tabular}


Table T1 (continued).

\begin{tabular}{|c|c|c|c|c|c|c|c|c|c|c|c|c|c|c|c|c|}
\hline \multirow{2}{*}{$\begin{array}{l}\text { Core, section, } \\
\text { interval }(\mathrm{cm})\end{array}$} & \multirow{2}{*}{$\begin{array}{l}\text { Depth } \\
\text { (mbsf) }\end{array}$} & \multirow{2}{*}{$\begin{array}{l}\text { Lith. } \\
\text { unit }\end{array}$} & \multicolumn{4}{|c|}{ X-ray diffraction peak area (total counts) } & \multirow{2}{*}{$\begin{array}{l}3.54 \AA / \\
3.58 \AA\end{array}$} & \multirow{2}{*}{\multicolumn{5}{|c|}{ Relative percent (Biscay factors) }} & \multicolumn{4}{|c|}{ Illite/smectite mixed-layer clay } \\
\hline & & & Smectite & Illite & Chlorite $(002)+$ & $1 / 8$ & & & & & & & $S(001)$ & $S(001)$ & Saddle/ & Percent \\
\hline $21 x$ & J & III & 173,6 & 4 & 27 & 13,857 & 2. & 27 & 29 & 30 & 11 & 2 & 4,322 & 7, & 0.59 & 67 \\
\hline $22 X-1,55-57$ & 179.45 & III & 49,875 & 72,519 & 159,794 & 20,036 & 3.84 & 7 & 43 & 37 & 10 & 3 & 3,248 & 4,015 & 0.81 & 57 \\
\hline
\end{tabular}

Note: $\mathrm{I} / \mathrm{V}=$ illite/vermiculite mixed layers. 